\title{
AN ASYMPTOTIC REPRESENTATION OF THE SAMPLE DISTRIBUTION FUNCTION
}

\author{
BY DAVID R. BRILLINGER
}

Communicated by David Blackwell, January 10, 1969

1. Let $X_{1}, \cdots, X_{n}$ be independent observations from the uniform distribution on $[0,1]$. Let $F_{n}(x)=$ the proportion of the $X_{j} \leqq x$. We will prove

THEOREM. There is a random function $\left\{G_{n}(x) ; 0 \leqq x \leqq 1\right\}$, with the same distribution as $\left\{F_{n}(x) ; 0 \leqq x \leqq 1\right\}$ for each $n$, and there is a Brownian motion $W$, such that for the Brownian $B(x)=n^{-1 / 2} W(n x)$

$$
\begin{aligned}
\sup _{0 \leqq x \leqq 1} \mid n^{1 / 2}\left[G_{n}(x)-x\right]-[B(x) & -x B(1)] \mid \\
& =O\left[n^{-1 / 4}(\log n)^{1 / 2}(\log \log n)^{1 / 4}\right]
\end{aligned}
$$

almost surely as $n \rightarrow \infty$.

This theorem is of use in the investigation of the asymptotic behavior of functionals of $\left\{F_{n}(x) ; 0 \leqq x \leqq 1\right\}$, especially functionals dependent on $\boldsymbol{n}$.

2. We construct $G_{n}(x)$ as follows; let $Y_{1}, Y_{2}, \ldots$ be independent exponential variables with mean 1 . Let $S(k)=Y_{1}+\cdots+Y_{k}, k$ $=1,2, \cdots$ and let $S(0)=0$. Set

$$
G_{n}(x)=k / n \quad \text { if } S(k) / S(n+1) \leqq x<S(k+1) / S(n+1) .
$$

This $\left\{G_{n}(x) ; 0 \leqq x \leqq 1\right\}$ has the same distribution as $\left\{F_{n}(x) ; 0 \leqq x \leqq 1\right\}$ for each $n$. We now record a series of lemmas.

LEMMA 1. There is a Brownian motion $W$ such that

$$
\sup _{1 \leqq k \leqq n}|k-S(k)-W(k)|=O\left[n^{1 / 4}(\log n)^{1 / 2}(\log \log n)^{1 / 4}\right]
$$

almost surely as $n \rightarrow \infty$.

Proof. This result is deducible from Theorem 1.5 of Strassen [8].

LEMMa 2. Almost surely as $n \rightarrow \infty$

$$
\sup _{0 \leqq x \leqq 1}\left|S\left(n G_{n}(x)\right)-x S(n+1)\right|=O\left[n^{1 / 4}\right] .
$$


Proof.

$$
\begin{aligned}
\mid S\left(n G_{n}(x)\right) & -x S(n+1) \mid & & \\
& =|S(k)-x S(n+1)| & & \text { if } S(k) \leqq x S(n+1)<S(k+1) \\
& \leqq S(k+1)-S(k) & & \text { if } S(k) \leqq x S(n+1)<S(k+1) . \\
& \leqq \max _{1 \leqq k \leqq n} Y_{k} & &
\end{aligned}
$$

and one sees, by elementary calculations, that this last $=O\left[n^{1 / 4}\right]$ almost surely as $n \rightarrow \infty$.

LEMMA 3. Almost surely as $n \rightarrow \infty$

$$
\begin{aligned}
\sup _{0 \leqq x \leqq 1} \mid n G_{n}(x)-S\left(n G_{n}(x)\right)-W & \left(n G_{n}(x)\right) \mid \\
& =O\left[n^{1 / 4}(\log n)^{1 / 2}(\log \log n)^{1 / 4}\right] .
\end{aligned}
$$

Proof.

$$
\begin{aligned}
\mid n G_{n}(x)- & S\left(n G_{n}(x)\right)-W\left(n G_{n}(x)\right) \mid \\
& =|k-S(k)-W(k)| \quad \text { if } S(k) \leqq x S(n+1)<S(k+1) \\
& \leqq \sup _{1 \leqq k \leqq n}|k-S(k)-W(k)|
\end{aligned}
$$

and (4) follows from (2).

LEMMA 4. Almost surely as $n \rightarrow \infty$

$$
\sup _{0 \leqq x \leqq 1}\left|G_{n}(x)-x\right|=O\left[n^{-1 / 2}(\log \log n)^{1 / 2}\right] .
$$

Proof. See Theorem 2* in Chung [3].

We next define the Brownian motion $B$ by $B(x)=n^{-1 / 2} W(n x)$ and then have

LEMMA 5. Almost surely as $n \rightarrow \infty$

$$
\text { (6) } \sup _{0 \leqq x \leqq 1}\left|B\left(G_{n}(x)\right)-B(x)\right|=O\left[n^{-1 / 4}(\log n)^{1 / 2}(\log \log n)^{1 / 4}\right] \text {. }
$$

Proof. (6) follows from (5) and Lévy's Hölder condition for Brownian motion (see Itô and Mcḱkean [4]) extended to apply to the interval $[0, n]$.

Proof of Theorem. Up to an error term

$$
O\left[n^{-1 / 4}(\log n)^{1 / 2}(\log \log n)^{1 / 4}\right],
$$

that is uniform in $x$, almost surely as $n \rightarrow \infty$ 


$$
\begin{aligned}
n^{1 / 2} G_{n}(x) & =n^{-1 / 2} S\left(n G_{n}(x)\right)+n^{-1 / 2} W\left(n G_{n}(x)\right) \quad \text { from (4), } \\
& =n^{-1 / 2} x S(n+1)+B\left(G_{n}(x)\right) \quad \text { from (3), } \\
& =n^{-1 / 2} x[(n+1)-W(n+1)]+B(x) \quad \text { from (2) and (6), } \\
& =n^{1 / 2} x-x B(1)+B(x),
\end{aligned}
$$

giving (1).

3. We may use the probability integral transformation to deduce a representation of the sample distribution function of observations from any continuous distribution. The results of Rosenkrantz [7] may be adapted to obtain rates of convergence in distribution for certain functionals of $F_{n}(x)$. The announcement of Kiefer [5] suggests that the error term in (1) may be best possible.

Bickel [1] and Billingsley [2] consider the weak convergence of the process $n^{1 / 2}\left[F_{n}(x)-x\right]$ to $W(x)-x W(1)$. Pyke and Root [6] let the distribution of $Y$ depend on $n$ and then prove

$$
\sup _{0 \leqq x \leqq 1}\left|n^{1 / 2}\left[G_{n}(x)-x\right]-[W(x)-x W(1)]\right|=o(1)
$$

almost surely as $n \rightarrow \infty$. I would like to thank Professor Pyke for the remark that $B$, as constructed above, depends on $n$.

\section{REFERENCES}

1. P. J. Bickel, Some contributions to the theory of order statistics, Proc. Fifth Berkeley Sympos. Math. Statist. and Probability (Berkeley, Calif., 1965/66), vol. I: Statistics, Univ. of California Press, Berkeley, Calif., 1967, pp. 575-591.

2. P. Billingsley, Convergence of probability measures, Wiley, New York, 1968.

3. K. L. Chung, An estimate concerning the Kolmogorov limit distribution, Trans. Amer. Math. Soc. 67 (1949), 36-50.

4. K. Ito and H. P. McKean, Jr., Diffusion processes and their sample paths, Springer-Verlag, Berlin, 1965.

5. J. Kiefer, The deviations in Skorokhod-Strassen approximation, Notices Amer. Math. Soc. 15 (1968), 936-937.

6. R. Pyke and D. Root, An application of stopping times to obtain weak convergence, Technical Report No. 16, University of Washington, Seattle, Wash., 1968.

7. W. A. Rosenkrantz, On rates of convergence for the invariance principle, Trans. Amer. Math. Soc. 129 (1967), 542-552.

8. V. Strassen, Almost sure behavior of sums of independent random variables and martingales, Proc. Fifth Berkeley Sympos. Math. Statist. and Probability (Berkeley, Calif., 1965/66), vol. II : Contributions to probability theory, Part 1, Univ. of California Press, Berkeley, Calif., 1967, pp. 315-343.

The London School of Economics and Political Science,

Aldwych, England 